\title{
The Clinical and Psychopathological Implications in the Forms of Hyperhistaminosis
}

\author{
Giulio Perrotta* \\ Psychologist sp.ing in Strategic Psychotherapy, Forensic Criminologist, Lawyer sp.ed SSPL, Researcher, Essayist, Italy
}

*Corresponding author: Giulio Perrotta, Psychologist sp.ing in Strategic Psychotherapy, Forensic Criminologist, Lawyer sp.ed SSPL, Researcher, Essayist, Italy

\begin{abstract}
The present work analyzes the clinical and psychopathological implications of hyperhistaminosis, starting from the general consideration that, despite the recent scarcity of research on the subject under examination, the correlation of hyperhistaminosis with different physical and psychic pathologies emerges and the direct relationship between body and mind and how these are interconnected. Hyperhistaminosis besides being involved in immune, neurodegenerative, systemic vascular, cardiac, pulmonary, hepatic, kidney and skin diseases, is directly connected to a large part of mood disorders, depression, obsessive disorder, phobias, and various personality disorders, even if it is not yet clear whether the role of histamine is the main factor or cause that exacerbates the symptoms, also taking into account the neurobiological and genetic correlates related to forms of hyperhistaminosis.
\end{abstract}

\section{Contents of the Manuscript}

\section{Histamine and the biological role in the human body}

Histamine or "2-(4-imidazolyl)ethylamine" (formula: C5H9N3) is an organic nitrogenous molecule belonging to the biogenic amine class, one of the chemical mediators of inflammation, and is derived from the decarboxylation of histidine by histidine decarboxylase [1]. The synthesis of histamine therefore starts with the decarboxylation of the amino acid "histidine" by a specific decarboxylase: the pyridoxal phosphate-dependent L-histidine decarboxylase. Catabolism, on the other hand, takes place through two metabolic pathways: oxidation is carried out by diamine oxidase which generates imidazole-acetic acid (IAA), while methylation carried out by histamine $\mathrm{N}$-methyltransferase gives tele- $\mathrm{N}$-methylhistamine ( $\mathrm{t}-\mathrm{MH}$ ) as a product. This in turn can be further metabolized by monoamine oxidase (MAO)-B, which generates tele-methyl methymidazol-acetic acid (t-MIAA). IAA, which is recognized as a GABA receptor agonist, also exists as a ribose and ribose-5-phosphate conjugate. Although IAA is formed in the brain by transamination of histidine, in some circumstances it can still be synthesized by histamine oxidation; however, histamine is a substrate of AED (but not of MAO) and in the central nervous system of vertebrates it is almost exclusively methylated.
Methylhistamine is then a substrate of MAO-B; therefore, MAO inhibitors increase the brain concentration of t-MH while lowering that of t-MIAA. In the brain, the concentrations of the latter are not affected by probenecid, an inhibitor of the transport of other metabolites derived from neurotransmitters [2].

In addition to the phenomenon of the resonance of the aromatic ring, histamine also gives the phenomenon of tautomeric. The molecule, therefore, exists in two very distinct forms in equilibrium between them, namely the tautomer $\tau$ (tau) and the tautomer $\pi$ (pi). In solution at physiological $\mathrm{pH}$, tautomer $\tau$ is more stable than tautomer $\pi$ because it is stabilized by the positive charge of the primary amine bound to the imidazole ring through the ethylene bridge. The receptor interaction is essentially based on the tautomeric phenomenon: while the $\mathrm{N} \tau-\mathrm{H}$ form has a high affinity for the receptor site, the $\mathrm{N} \pi-\mathrm{H}$ form has high efficacy, i.e. it is the tautomeric form able to activate the response. Histamine, therefore, binds to the receptor when it is in the $\mathrm{N} \tau-\mathrm{H}$ form with the primary protonated amine (positively charged), the interaction of the amine with the anionic pocket of the active receptor site neutralizes the positive charge of histamine thus stabilizing the $\mathrm{N} \pi-\mathrm{H}$ form, a tautomeric form with low affinity but capable of activating the intracellular response [3,4] (Figure 1). 


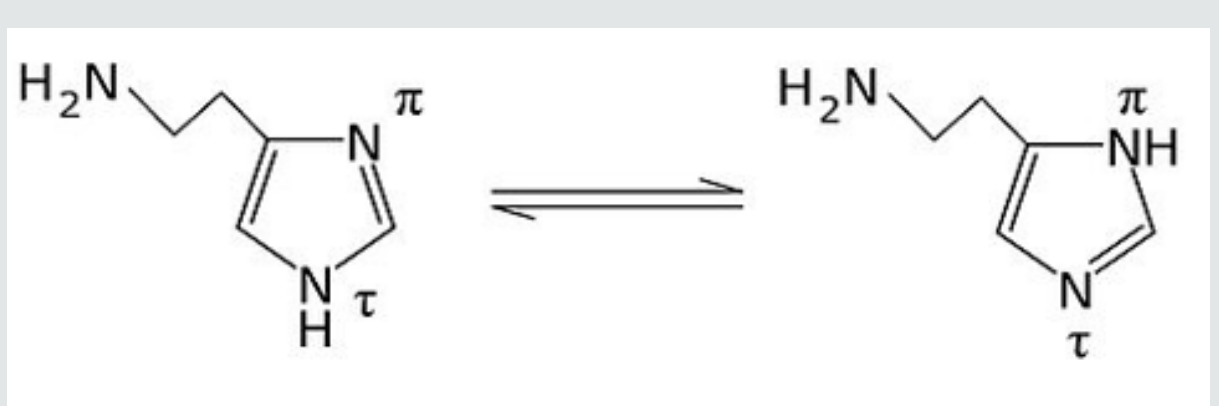

Figure 1: The two distinct forms of the histamine molecule.

Histamine is stored in specialized cells such as eosinophilic granulocytes, platelets, mast cells, the first two circulating in the blood, the last one interspersed in the connective tissue around blood vessels, in the skin and mucous membranes of the digestive and respiratory tract. Histamine is contained in the delta granules of platelets, mast cells, and basophils. Mast cells are cells resident in connective tissue that have IgE receptors on the surface, immunoglobulins released into the body following an allergic reaction. The receptors on the surface of mast cells bind to the circulating IgE and this complex binds to the allergen (which triggered the reaction). This attack causes a series of reactions within the mast cells, with the final release of many substances, including histamine [5] (Figure 2).

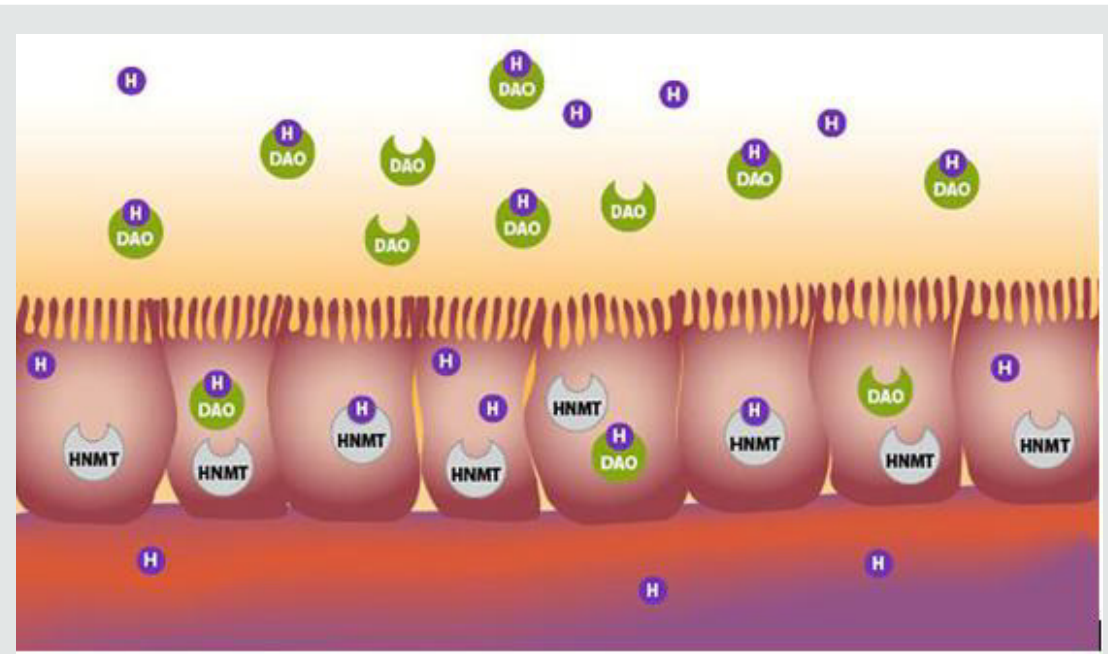

Figure 2: Most of the histamine we consume is neutralized by AED released in the gastrointestinal tract or by HNMT found in the cells lining the intestine. These enzymes prevent excess histamine from entering the bloodstream. [Illustration by Suzi Smith; Adapted from Kovacova-Hanuskova 2015 Allergol Immunopathol (Madr) 43 (5): 498-506].

Histamine [6], in the human body, mainly causes:

a) Vasoconstriction of the great arteries, by action on the smooth muscles (hypertension);

b) Vasodilation of the arterioles, an effect that is normally carried out locally. If, however, vasodilation occurs at the systemic level, there is hypotension.

c) Increased permeability of the capillaries and postcapillary venules, through various modifications of the endothelial cells. Acting in this way allows a greater influx of leukocytes into the traumatized and possibly infected area;

d) Bronchoconstriction due to the contraction of bronchial muscle cells. Histamine is chronologically one of the first mediators involved in the development of the symptoms of an allergic asthma attack.

The effect of histamine, which accompanies vasodilation, is to alter the balance between the liquid component of the blood and the water contained in the connective tissue: the result is an accumulation of liquids in the connective tissue that causes edema. Edema can be a serious risk if it occurs in the throat or bronchi. A second exposure to the allergen can have very violent effects because in that case, IgE antibodies (immunoglobulins) are already present on the mast cell [7].

The effects of histamine [1,8-10] are mediated in different tissues by four specific receptors, which are a class of sevensegment transmembrane receptors bound to $G$ proteins, which 
have histamine as an "endogenous ligand" (a molecule capable of binding a biomolecule and forming a complex capable of performing biological functions):

a) H1: H1 receptors are coupled to Gq proteins and act with an increase in intracellular calcium by activation of phospholipase C. Their activation by histamine or histamine-mimetic substances causes vasodilation, although there is an increase in intracellular calcium, because they are not located at the level of smooth myocells but the level of endothelial cells. Histamine causes an increase in calcium which activates the nitrous oxide-synthase with the formation of nitroxide (NO) which acts as a mediator because it spreads in the muscle layer where it activates guanylatecyclase with the formation of cGMP and consequent vasodilation. Guanylate-cyclase has a heme group in its structure that receives $\mathrm{NO}$ and is also soluble at the cytoplasmic level. In addition to the endothelial cells lining the small-caliber vessels, especially the postcapillary venules, these receptors are found in intestine, bronchi, cardiovascular system, central nervous system (postsynaptic localization). The effects are contraction of the smooth bronchial muscles, vasodilation, increased capillary permeability, and maintenance of wakefulness. They are coupled to a Gq and G11 protein which acts through the hydrolysis of membrane phosphoinositide (intervention of a phospholipase C), generating diacylglycerol (DAG) and inositol triphosphate (IP3), which set in motion calcium-dependent transductive pathways.

b) H2: they are coupled with Gs proteins with consequent activation of adenylate-cyclase, cAMP formation, activation of protein kinase A and increase in intracytoplasmic calcium. They are mainly found in the gastric wall, the immune system, and the cardiovascular system. The effects are increased acid secretion of the stomach and vasodilation. They activate adenylatocyclase via a Gs protein and therefore their molecular actions are mediated by cyclic MPA and protein kinase A (PKA). The vasodilatative effect occurs thanks to the consequent inactivation (by phosphorylation) of the light chain myosin kinase (MLCK): myosin can no longer bind to actin and contraction is avoided.

c) H3: are presynaptic auto receptors in the central nervous system and are coupled to Gs proteins with consequent activation of adenylate-cyclase, cAMP formation, activation of protein kinase $A$, and increase of intracytoplasmic calcium. They are mainly found in the central nervous system and are presynaptic auto receptors designed to control biosynthesis and the release of histamine and other neurotransmitters. In particular, histamine acting on these receptors has an inhibiting action on the release of neurotransmitters associated with that synapse. Their effect is expressed through an inhibitory G protein (Gi); therefore, there is a reduction in the activity of adenylatocyclase; beta-gamma dimers can lead to the activation or inhibition of certain ion channels. Through this mechanism, H3 receptors control the secretion of certain neurotransmitters.

d) H4: Recently discovered, the molecular mechanism is not yet clear. They are mainly found in bone marrow, spleen, eosinophils, mast cells, neutrophils, and T-helper lymphocytes. The effects are to modulate the activation of the immune response. These receptors also inhibit adenylatocyclase via a Gi. They can also mate with a G-zero (G0), which regulates potassium channels and the activation of MAP-kinases via PKC. It has been reported that they can also activate $G$ proteins present exclusively in marrow cells, G15 and G16, through which they can act on the mobilization of calcium from intracellular deposits (Figure 3).

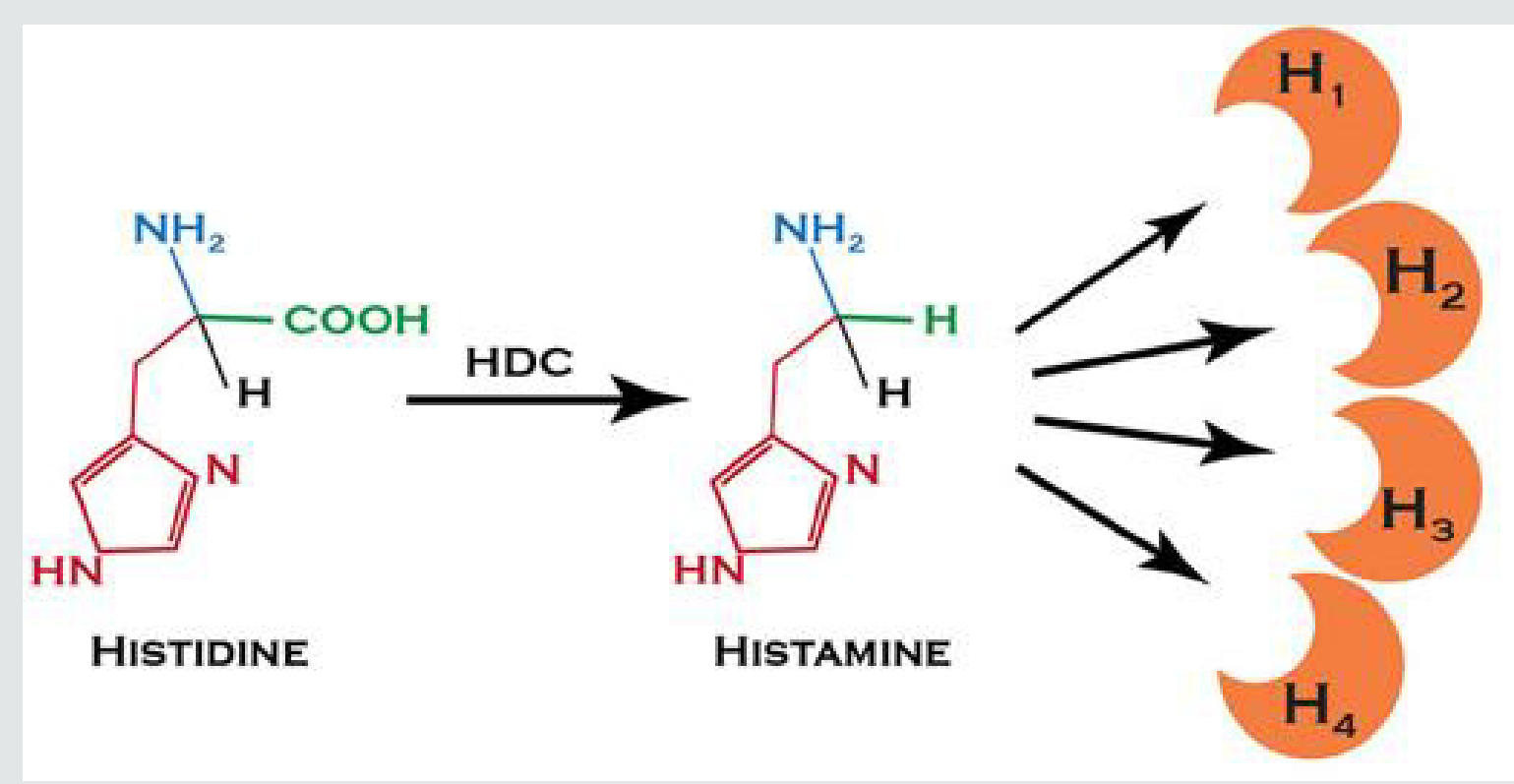

Figure 3: The four histaminic receptors. 
Most histamine release is non-synaptic but neuronal histamine may also be capable of fast neurotransmission; for example, histamine increases chlorine ion conductance in the thalamus through its $\mathrm{H} 2$ receptors or an ion channel and this implies that, like serotonin, histamine can activate both second messenger receptors and ligand-controlled ion channels [1].

Histaminergic neurons can also regulate and be regulated by other neurochemical pathways; for example, the activation of $\mathrm{H} 3$ auto receptors reduces the release of acetylcholine, noradrenaline, serotonin, and certain peptides. In turn, activation of the NMDA receptor, mu-opioid receptors, D2 dopaminergic, and serotonin receptor subtypes may increase neuronal histamine release. Of particular interest is the role of the cholinergic system in histamineinduced waking. The appearance of sleep has been traced in the cells of the ventral preoptic area of the hypothalamus which, when activated, inhibits tube-mammillary neurons through GABA discharges. So, histamine is an important regulator of sleep-wake cycles. It is on this basis that the side effects of certain anti-allergic drugs (drowsiness) are explained, thanks to the antagonism they have towards the $\mathrm{H} 1$ receptors in the brain. Another effect also mediated by $\mathrm{H} 1$ receptors is the suppression of convulsive activity [9].

At the hypothalamic level histamine is a regulator of other functions, such as the release of vasopressin, oxytocin, prolactin, $\mathrm{ACTH}$, and beta-endorphin. It also regulates hunger and thirst: an increase in histamine suppresses these two sensations, while $\mathrm{H} 1$ receptor antagonists act on the ventromedial hypothalamus to stimulate appetite. At the pituitary level, it seems that both $\mathrm{H} 1$ and $\mathrm{H} 2$ receptors are implicated in the regulation of local hormones. In the experimental animal, histamine, after microinjection in certain areas of the brain, is also an effective suppressor of painful responses, an effect that seems to be mainly mediated by $\mathrm{H} 2$ receptors. Outside the brain, the presence of $\mathrm{H} 1$ and $\mathrm{H} 3$ receptors on certain types of sensory nerves promote prophlogistic/nociceptive and antiphlogistic/nociceptive responses, respectively. Neuronal histamine is also a mediator of the condition known as kinetosis; histaminergic neurons are activated by vestibular disorders and thus secrete histamine to the centers of brainstem vomiting (in fact, $\mathrm{H} 1$ receptor antagonists are generally good antiemetics) [1,5] (Figure 4).

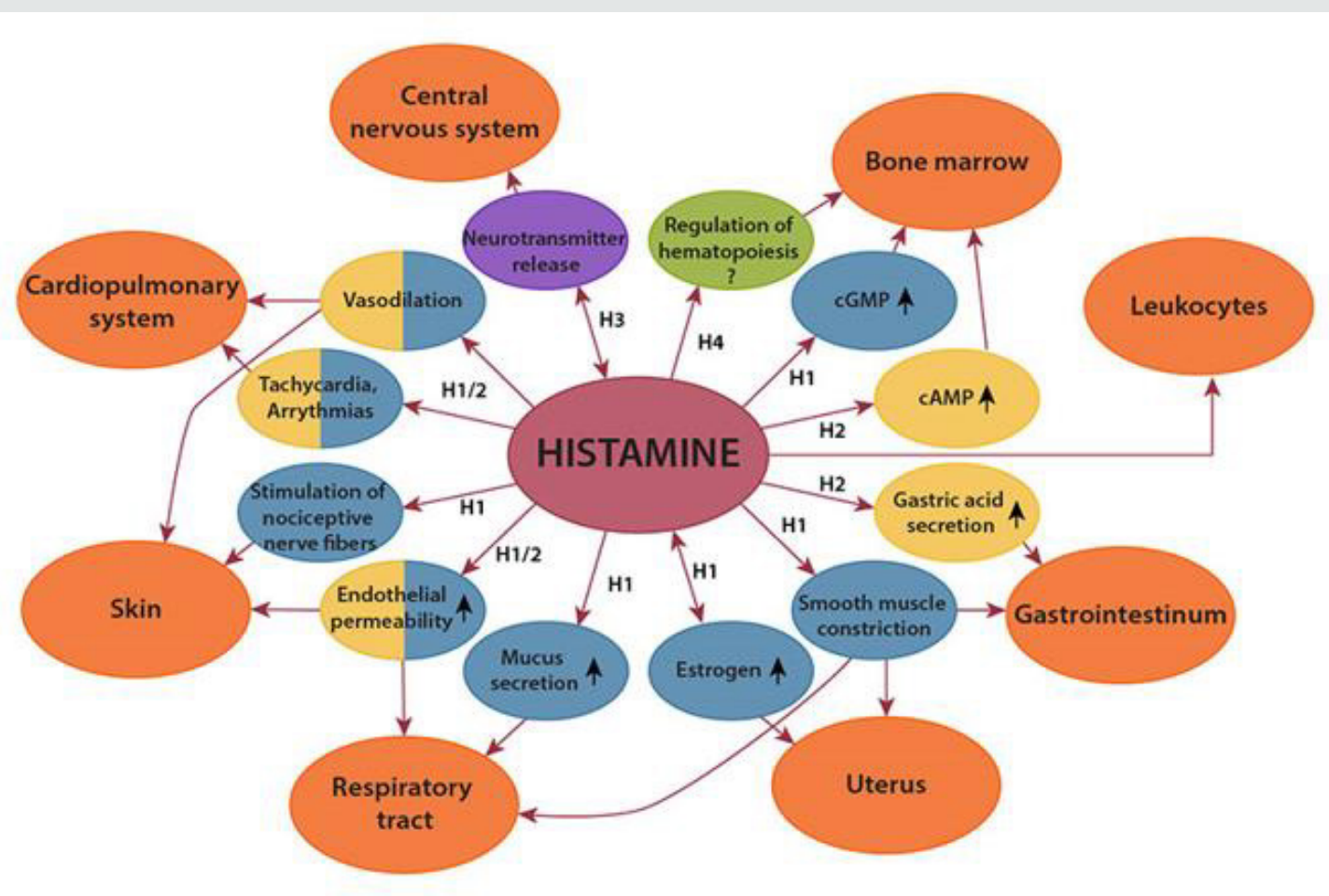

Figure 4: Summary diagram of histamine functions (Maintz 2007, Am J Clin Nutr 85: 1185-1196).

\section{Monoamine Oxidase Inhibitors and the Role in Neurological and Psychopathological Conditions}

Monoamine oxidase inhibitors (MAO-I) are a class of drugs that can reduce or block the activity of monoamine oxidases, the enzymes that oxidatively metabolize monoamines. Monoamines include numerous endogenous substances such as some neurotransmitters (such as serotonin and catecholamines such as adrenaline, noradrenaline, melatonin, and dopamine) and exogenous compounds (such as tyramine and some drugs) [11].

MAO-I block the activity of monoaminoxidase enzymes, the mitochondrial enzymes that catalyze the oxidative deamination reaction of endogenous and exogenous amines. There are two 
isoforms of monoaminoxidase: MAO-A which preferentially degrade serotonin, melatonin, noradrenaline, adrenaline, dopamine, and tryptamine; MAO-B which degrade dopamine, tryptamine, and phenylethylamine. Both isoforms are present in the central nervous system where they are among the main responsible for the degradation of monoamine neurotransmitters, however, MAO-A is also extensively expressed in the liver (where they degrade monoamines taken with the diet such as tyramine), in the gastrointestinal tract, in the placenta, and the pulmonary vascular endothelium, while MAO-B are found mainly in platelets [12].

The inhibition of MAOs at the central nervous system level [13] leads to an increase in both intracellular and synaptic levels of catecholamine neurotransmitters and serotonin, which play a role in psychiatric disorders. The blockage can be of two types:

a) "irreversible", such as that caused by traditional MAO-I, which causes the enzyme to be irreversibly inactivated and then re-synthesized by the cell. It takes the cell approximately 2 weeks to regenerate the monoamine oxidase stockpile after the discontinuation of the drug and for this reason, it should wait at least 2 weeks after the discontinuation before taking drugs that can interact with MAO-I.

b) "reversible", which does not inactivate the enzyme irreversibly but retains partial activity and can be removed by exogenous amines such as tyramine. The drugs belonging to this class are called reversible inhibitors of monoaminoxidase (or RIMA) and thanks to this reversibility they do not interact with tyramine or other sympathomimetic amines. They include toloxatone and moclobemide and some compounds contained in natural extracts such as harmaline.

The different drugs may be more or less selective towards one of the two isoforms (e.g. MAOA are mainly inhibited by clorgyline while MAOB is selectively inhibited by selegiline) or lack selectivity (as in the case of phenelzine or tranylcypromine). The compounds that irreversibly inhibit MAO-A, the form that preferentially degrades serotonin and tyramine, are those with the greatest potential for interaction with foods containing tyramine (cured foods such as sausages and some cheeses), which can result in hypertensive crises, and serotonergic agents (such as some antidepressants), which can result in serotonergic syndrome, which in some cases are fatal. These restrictions do not apply to selective MAO-B inhibitors, reversible and transdermal formulations that skip liver metabolism and do not require dietary restrictions [12].

The inhibition of the degradation of neurotransmitters $[12,13]$, which are important in the regulation of mood tone, leads to their increase in the central nervous system: this mechanism is responsible for the antidepressant and anxiolytic effect of MAO-I. In particular: a) The non-selective inhibitors of the $\mathrm{A}$ and $\mathrm{B}$ isoforms or the selective ones for form A of MAO-I only are used today as antidepressants (they were the first antidepressants to be marketed) especially for forms resistant to treatment with firstline drugs and in the case of atypical depression, where they show high efficacy.

b) Isoform B inhibitors alone are used in the early stages of Parkinson's disease.

c) In the treatment of depression and other psychiatric disorders, although highly effective and tolerable, often superior to other classes of drugs (including SSRIs), irreversible MAO-I is considered a second choice because of potentially severe drug and dietary interactions requiring a diet of tyramine-free foods.

d) Monoamine oxidase inhibitors have been found effective in the treatment of panic disorder, including when associated with agoraphobia or social phobia, in the treatment of dysthymia, atypical depression, major depression, and bipolar depression, in the treatment of generalized anxiety, bulimia, obsessive-compulsive disorder, post-traumatic stress disorder, personality avoidance disorder, and borderline personality disorder.

The class MAO-I [14] is divided as follows:

1) Hydrazinic derivatives:

a) hydrazinic substitutes: iproniazid, phenelzine

b) hydrazinic derivatives as such: isoniazid

2) Non-hydrazine derivatives:

c) certain $\beta$-carbolins

d) Harmala alkaloids: armin, armaline, tetrahydroarmine

e) indolalkylamines: methyltryptamine

f) propargylamines: chlorgiline, pargyline, selegiline

g) cyclopropropylamines: tranylcypromine

Pharmacologically [15], however:

1) Non-selective inhibitors MAO-a, MAO-b:

a) Hydrazine

b) Hydralazine

c) Iproclozide

d) Iproniazid

e) Isocarboxazide 
f) Isoniazide

g) Mebanazine

h) Nialamide

i) Phenelzine

j) Pheniprazine

k) Procarbazine

l) Non-hydrazines

m) Caroxazone

n) Furazolidone

o) Linezolid

p) Tranylcypromine

2) Selective MAO-a Inhibitors:

a) Chlorgiline

b) Minaprine

c) Moclobemide

d) Toloxatone

3) Selective MAO-b Inhibitors:

a) Lazabemide

b) Pargyline

c) Rasagiline

d) Selegiline

4) Natural derivatives:

a) Non-selective inhibitors MAO-a, MAO-b: Curcumin and Harmala alkaloids (armalin, armaline, tetrahydroarmine)

b) MAO-inhibitor Selective: Berberine, Coptisin, Resveratrol

c) Selective MAO-b Inhibitors: Catechin, Hydroxytyrosol, Piperin

d) Unknown selectivity: Licorice and Siberian Ginseng.

When administered orally, MAO-I is rapidly absorbed by the digestive tract. Appropriate MAO inhibition is achieved in 5-10 days, depending on the drug used. The cessation of all pharmacological effects occurs within 15-20 days after discontinuation of treatment, when all inactive MAOIs are metabolized and replaced by new ones. Taking MAO-I inhibits the catabolism of catecholamines, serotonin, and other exogenous sympathomimetic amines (including some drugs and tyramine), greatly increasing their amounts; these neurotransmitters in the bloodstream play various roles in regulating circulatory function. The combined intake of MAO-I with certain drugs or foods containing tyramine can cause hypertensive crises, even severe ones, which initially manifest themselves with a pulsating occipital headache with frontal irradiation, palpitations, hypertension, nausea, stiffness and neck pain and which can very rarely evolve into intracranial hemorrhage and hemiplegia which in very rare cases have had fatal results. Therefore, the intake of MAO-I should not be accompanied by sympathomimetic drugs (adrenaline, ephedrine) and a particular dietary restriction should also be applied: the intake of MAO-I combined with foods containing a high amount of tyramine (mature foods such as some cheeses, sausages, some spirits) can trigger an excessive release of norepinephrine which can lead to a hypertensive crisis. MAO-I may be associated with other psychoactive substances only under the strict supervision of a licensed therapist. Finally, dietary restrictions do not apply to newer formulations of these drugs such as moclobemide (a reversible MAO-I inhibitor that allows degradation of exogenous catecholamines) and transdermal selegiline patches (which skip liver metabolism and allow degradation of tyramine and other amines taken with the diet) [14].

\section{Clinical Conditions Resulting from Hyperhistaminosis}

Significant changes in the histaminergic system have been found in some neurological disorders such as multiple sclerosis, Wernicke's alcoholic encephalopathy, Alzheimer's disease, Parkinson's disease, and Down syndrome [16]. Whether derived from mast cells or neurons, histamine can participate in these processes by controlling vascular permeability and the blood-brain barrier to peripheral immune cells [5]. The ability of histamine to enhance NMDA-dependent responses could explain some of its neurotoxic effects found in toxic encephalopathies, especially those from alcohol and chronic hepatopathies (ammoniopathies), but also some genetic conditions such as histidinemia, where histamine synthesis receives severe imbalances [17].

But without a doubt, the most common condition [18] is determined by the increasing histamine disorder which leads to an exponential increase in allergic symptoms. Healthy individuals should be able to manage foods containing 50 to $100 \mathrm{mg} / \mathrm{kg}$ of histamine, but people with histamine intolerance generally react at much lower levels. The types of reactions that can occur mainly depend on how much histamine we are exposed to and what area of the body is affected. Reactions can be divided into five general categories:

a) anaphylaxis-massage, which releases histamine into the bloodstream as a result of an allergic reaction.

b) mackerel poisoning, caused by eating cheese, meat and damaged fish (especially bluefish, tuna, mackerel, salmon, sole, plaice, swordfish, herring, sardines, anchovies, and non-glass canned fish), which contains more than $500 \mathrm{mg} / \mathrm{kg}$ histamine;

c) localized allergic reactions; 
d) histamine overload, when exaggerating with histaminerich foods;

e) histamine intolerance, which occurs only in sensitive persons already at dosages below $50 \mathrm{mg} / \mathrm{kg}$.

The term has been introduced as a common denominator for symptoms such as abdominal pain, flatulence, diarrhea, headache (itching), blepharedema (swollen eyes), urticaria (hives), rhinorrhoea (runny nose), dysmenorrhoea (problems related to the menstrual cycle), respiratory obstruction (difficulty breathing), tachycardia (heartbeat), extrasystoles (palpitations), and hypotension (low blood pressure) that occur after eating histamine-rich foods. Most cases of intolerance are thought to be caused by abnormally low levels of AED quantity or activity, due to: genetic defects (as in the case of the AOC1 gene, with the variants "c.47C>T", "c.995C>T" and "c.1990 C>G"), structural and functional damage to the small intestine, ascending colon, kidneys, liver, placenta (during pregnancy, symptoms of AED deficiency regress) and still those who undergo chemotherapy, suffer from inflamed bowel and have systemic-inflammatory syndromes such as SIBO and Leaky GUT. However, an inflamed intestine will have much less synthesis capacity than an intact intestine. AED also requires vitamin B6, B9, and B12, vitamin C, copper, and zinc to function properly. Some drugs can also reduce the production of the enzyme AED, such as acetylcysteine, ambroxol, aminophylline, amitriptyline, chloroquine, isoniazid, methimazole, metoclopramide, propafenone, and verapamil.
Female histamine intolerants are frequently seen in the clinic; this is because estrogen and histamine reinforce each other, which means that estrogen increases histamine levels and vice versa. Since women have higher levels of estrogen than men, they consequently have higher levels of histamine than men. It is therefore obvious that smaller amounts of histamine-high histamine foods are enough to overwhelm histamine-destroying enzymes. Histamine is known to increase estrogen production. This is a very important factor to think about, because ingesting large amounts of histamine overnight can put women on a hormonal roller coaster due to fluctuating estrogen levels. Women may be more sensitive to histamine in food at various points in the cycle when estrogen levels are higher, i.e. up to ovulation, or if they are taking estrogen supplements. Histamine causes the release of nitric oxide into the arteries surrounding the brain, which leads to headaches and lays the foundation for the famous "menstrual migraines". Women have more H1 receptors in their brains than men, which means that women's brains are naturally more sensitive to histamine (see mental health section below). One of the many natural roles of histamine in the body is to stimulate the contraction of the uterus. This explains why histamine intolerance can cause or worsen pre-menstrual cramps. Above all, it is fascinating that pregnant women generally feel less affected by allergies and intolerances, because the placenta produces up to 500 times the normal amount of AED, the enzyme that destroys histamine, to protect the fetus from histamine toxicity. It would be very interesting to investigate the estrogen-histamine link in the light of pathologies such as polycystic ovary [19] (Figure 5).

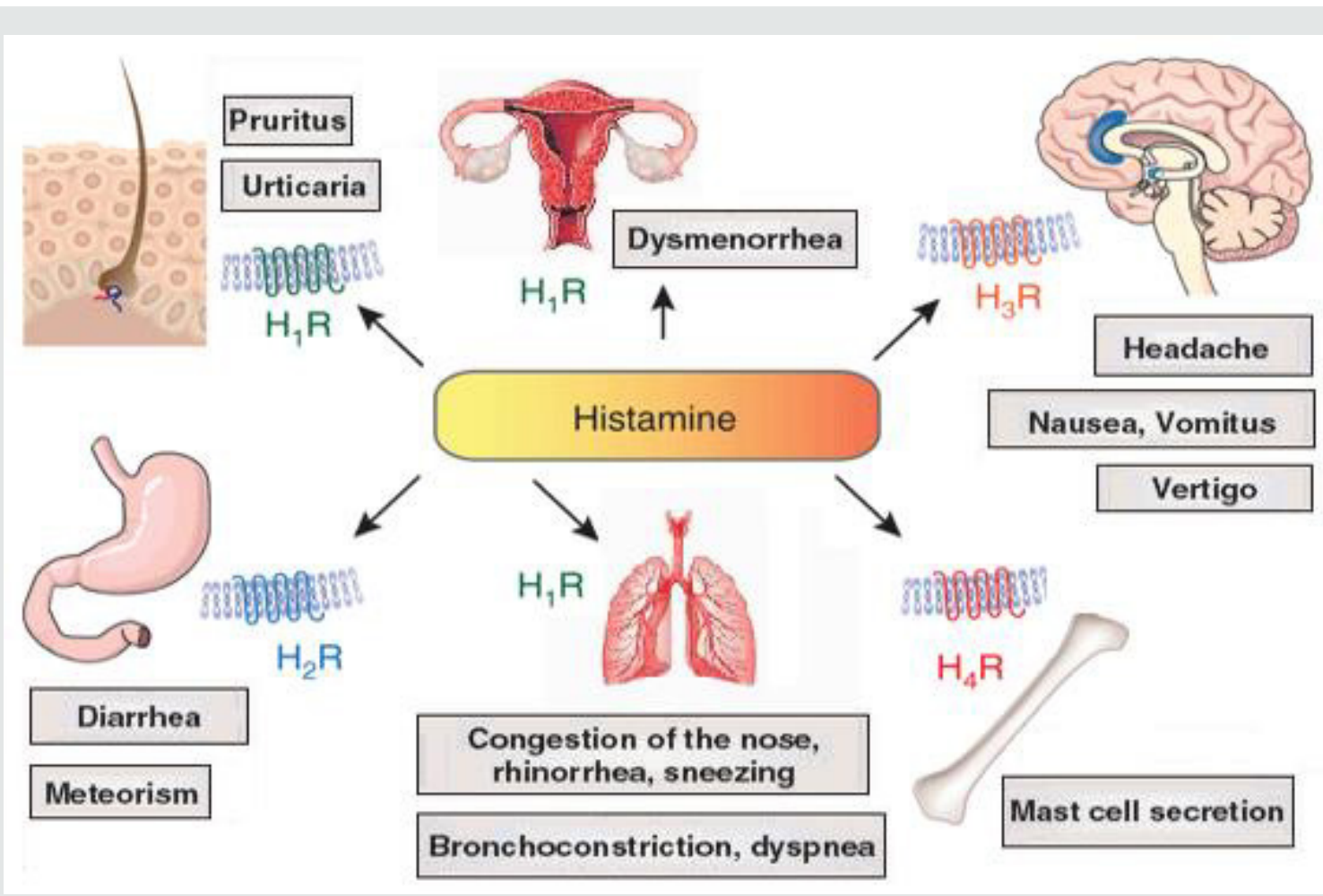

Figure 5: Summary diagram of symptoms following histamine intolerance. 
If, on the contrary, the release of histamine is greater than the amount of enzyme capable of catabolizing it, as a result of excessive production of IgE (immunoglobulin E), in that case, you will have to search for the origin of the morbid condition in another disorder: Hyper-IGE Syndrome" (or Job's Syndrome), a primitive immunodeficiency characterized by high serum IgE levels (> $2000 \mathrm{IU} / \mathrm{ml}$ ), recurrent staphylococcal abscesses and recurrent pneumonia associated with pneumatocele formation. However, if IgE production is lower, it is possible to speak of a hyper-IgE induced by the high amount of histamine circulating in the serum [20].

Immunoglobulin E (IgE) are antibodies produced by the immune system in response to a stimulus perceived as a threat. They correspond to one of five classes of immunoglobulins (G, A, $\mathrm{M}, \mathrm{D}$, and $\mathrm{E}$ ) and are normally present in the bloodstream in very low concentrations. This test measures the concentration of IgE in the blood. Immunoglobulins are essential proteins for the proper functioning of the body's immune system. They are produced by specific immune cells called plasma cells and attack bacteria, viruses, other microorganisms, and substances recognized as "foreign" and potentially harmful to the immune system. The production of IgE can lead to the manifestation of allergic reactions such as asthma but is also implicated in the immune response against parasites. In allergy sufferers, the body reacts excessively to one or more substances in the environment, called allergens, which in other individuals may not cause any reaction. Allergy can develop as a result of exposure to a substance such as pollen, peanuts, eggs, strawberries, bee venom, and hundreds of others. During the first exposure, called sensitization, the allergy sufferer produces specific IgE directed against the allergen. The IgE produced bind specialized white blood cells causing the release of certain substances, including histamine, during subsequent exposures. In allergic/ asthmatic people these substances can cause lung bronchial constriction or other reactions including runny nose, eye, and skin itching. Whenever the allergy sufferer is exposed to allergens to which he or she has become sensitized during the first exposure, IgE is produced rapidly, increasing until the allergic reaction is triggered. The severity of the reaction and the symptoms associated with it can range from localized redness and itching of the skin to respiratory failure, vomiting, diarrhea, and, in some cases, lifethreatening anaphylactic shock. The severity of the reaction varies from person to person, episode to episode and may worsen over time [7].

Hyper-IgE Syndromes (HIES) [21-30] include a group of primary immunodeficiency disorders characterized by high serum IgE levels, recurrent skin abscesses caused by Staphylococcus aureus, eczema, hypereosinophilia, and lung infections. Both an autosomal dominant (AD-HIES) and recessive (AR-HIES) form of this disorder is described: a) AD-HIES. There is a heterozygous mutation of the STAT3 gene (signal transducer and activator of transcription 3) that promotes the transcription of target genes and modifies the epigenetic status of chromatin, being fundamental for cytokine development, differentiation, and survival. STAT-3 appears to have a predominant role in the differentiation of Th17 lymphocytes by cytokines such as IL- 6 and IL-23. Therefore, the reduction of Th17 lymphocytes is a common hallmark of $\mathrm{AD}$-HIES and a reduced differentiation of these lymphocytes will lead to a lack of production of IL-22 and IL-17. The latter are fundamental cytokines for the synthesis of antimicrobial peptides and chemokines involved in the immune response against fungi, which easily infect the respiratory tract and mucous membranes. Patients with AD-HIES are also deficient in the antibody response due to the functional reduction of antigen-specific memory $\mathrm{B}$ lymphocytes. This appears to be related to the signal of IL-21 and IL-10 which induce the differentiation of B cells into secretive plasma cells. Follicular $\mathrm{T}$ helper lymphocytes, in turn, reduced due to a deficit in differentiation, induce $\mathrm{B}$ lymphocytes to differentiate into memory cells. Although all affected subjects present a high number of circulating IgE and a marked blood eosinophilia, there is no imbalance towards a Th2 phenotype and therefore show a reduced rate of allergic or anaphylaxis related diseases.

b) AR-HIES. The gene mutation responsible for AR-HIES, identified in the DOCK8 gene, induces the rearrangement of actin filaments, and regulates cell growth, adhesion, and migration in the formation of the immunological synapse. The lymphocytic proliferation stimulated by mitogens is reduced, although several cell lines are numerically in the norm for a possible qualitative defect of the $\mathrm{T}$ line. Only a few years ago, other recessive mutations have been identified that may be responsible for pathological patterns similar to Hyper IgE syndromes. Worth mentioning is the mutation of Tyk2 (Tyrosine Kinase 2), associated with IFN $\gamma$ and IL-12 signaling. More recently, in 2015, a new mutation has been identified as the cause of a recessive form of HIES. This mutation involves the phosphoglucomutase 3 (PGM3) gene.

The therapeutic approach of Hyper IgE syndromes is based on a correct management of respiratory and skin infections, through aggressive antibiotic and antifungal treatments. Skin infections should be managed to take into account the marked skin colonization by S. aureus; while in the respiratory tract the eradication of pathogens such as Aspergillus or Candida which tends to colonize even the gold-pharyngeal mucous membranes. A critical point is represented by post-infectious complications of the pulmonary parenchyma, mainly after infections by mycobacteria, Pseudomonas, and Aspergillus. The surgical approach may be associated with additional risks of post-operative outcomes due to 
the defective scarring system of these patients. Also, to be considered in the management of hypertension, in some cases high-dose Ig ev replacement therapy may be considered, although the data on this subject are discordant. Hematopoietic stem cell transplantation could represent a promising therapeutic option according to initial data, also considering the high mortality and morbidity rate associated with this pathology. However, the first case of marrow transplantation in the HIES reported in the literature was not able to prevent infectious episodes or to reduce IgE levels (unchanged); the patient subsequently died due to lymphoma. However, in a second report, the two hematopoietic stem cell transplant patients showed normalized IL-17 levels and reduced IgE levels in the follow-up, as well as a decreased frequency of respiratory and skin infections (particularly caused by Staphylococcus and Candida). However, long-term lung complications (pneumatocele, bronchiectasis) and bone fragility persisted [31-35].

\section{The Psychopathological Implications in Hyper histamine Hypotheses}

Although histamine molecules are found in both the body and the brain, they do not cross the blood-brain barrier; however, histamine intolerance can still affect mental health [36]. Histamine also acts as an important neurotransmitter in the brain, where it helps regulate stress response, alertness, sexual function, sleepwake cycles, attention, learning, and memory. Although there are no direct correlations and studies with representative samples that specifically demonstrate the formal link between histamine intolerance and mood disorders, people with histamine intolerance commonly report symptoms of easy fatigue, hypersensitivity to heat, anxiety, panic, phobia, and insomnia. We know that histamine is not able to cross the blood-brain barrier, however, it can interfere with other hormones that affect the nervous system, such as catecholamines; even adrenaline does not cross the blood-brain barrier, but in the body, it can trigger symptoms of panic, heart in the throat, anxiety, nausea, sweating, insomnia, shortness of breath, tremors, and other typical symptoms.

Another interesting hypothesis is the correlation with estrogen, which can interfere with testosterone, affecting plasma levels: once inside the brain, estrogen stimulates the release of histamine, thus creating another vicious circle, more histamine, more estrogen, and more histamine. In the brain, histamine is not degraded by the enzyme DAO (as it is not expressed in the brain) but by the enzyme HNMT, which belongs to the class of transferases. HNMT catalyzes the movement of a methyl group from SAM, which is one of the major donors of methyl groups in our metabolism, to histamine, transforming it into $\mathrm{N}$-amethystine; however, if you are a carrier of MHTFR polymorphisms, the production of SAM-E will be significantly reduced, and with it the availability of substrate necessary for the degradation of histamine itself. In the brainstem, we have two structures that are particularly related to the waking state, the reticular formation, and the pretectal nuclei. Our eyes have a connection with both these systems for a very simple reason, the light that reaches our eyes is the main regulator of the sleep-wake rhythm, the light, especially the frequency attributable to the blue of the sky during the day through the stimulation of these nuclei promotes a general activation of the whole central nervous system. In the end, it is quite intuitive, and it is an ancestral memory of what has always been our life on this planet, with the sun we wake up, with the night we sleep. Unfortunately, now the artificial light, televisions, smartphones, PC monitors, we find ourselves bombarded by artificial radiation that promotes a hyperactivation of the central nervous system when unwanted and unnecessary. The mamillar bodies that are specific gray nuclei connected to the hypothalamus synthesize histamine, which works as a neurotransmitter. Let us remember that the hypothalamus is a component of the central nervous system that regulates pituitary release, controls the whole autonomic nervous system, and is also strongly involved in the limbic system. The hypothalamic impact on the metabolism is mastodon tic. The histamine that is released has a systemic effect on the brain, it reaches practically all the various components of the central nervous system, from the spinal cord, the cerebellum, the base nuclei, the cerebral cortex to the nuclei of the cranial nerves, especially the vestibular ones that control the balance. Together with cortisol, histamine is part of the body's natural response to stress. The problem arises when we have a dysregulation of histamine metabolism. This is a very important point, an accumulation of histamine in the brain is seen as a sign of imminent danger, leading to the activation of all stress-related pathways, even if there are no real causes for being under stress. Histamine, therefore, becomes an important factor in the release of chronic and out-of-rhythm circadian cortisol that leads to all those situations of chronic cortisol excess. The H3R and H1R receptors regulate the sympathetic autonomic nervous system and promote the release of cortisol by the adrenals. Phenomena of adrenal fatigue are quite common in people intolerant to histamine. Histamine promotes the release of estrogen, which in turn stimulates the release of histamine by mast cells. It is a vicious circle, often histamine-intolerant women suffer from estrogen dominance. Histamine is also linked to the release of TSH, the H2R receptor decreases the release of TRH, which stimulates TSH and therefore the release of $\mathrm{T} 3$ and $\mathrm{T} 4$. Often histamine intolerants are hypothyroid non-autoimmune. H3R receptor antagonists decrease appetite, make people lose weight, while H1R antagonists stimulate appetite and fattening. It is quite normal to see histamine intolerant people who have problems with weight, whether it is difficult to put it on or lose it. Histamine intolerance is also implicated in addictions and compulsive behavior. Especially the H2R receptor is implicated in the mechanisms of rewarding pleasure, memory, learning. It seems that various addictive substances such as sugar, psychotropic drugs, drugs, alcohol interfere with the metabolism 
of histamine in the brain. Binge eating, anorexia, bulimia are linked to the reward mechanisms of the $\mathrm{H} 2 \mathrm{R}$ receptor, they are coming on the market of drugs against appetite disorders acting on the H3R receptor. The metabolic syndrome is another consequence of bad histamine metabolism. The release of histamine from the brain regulates leptin, which in turn regulates satiety and obesity. Histamine also seems to be strongly related to insulin resistance. The H1R receptor promotes a brain excitement that often results in anxiety and underlying nervousness. Histamine regulates pain perception, H1R receptor activation increases pain perception, while $\mathrm{H} 2 \mathrm{R}$ receptor activation decreases it. Since itching follows the same pathways as pain, the sensation of itching is also modulated by the activation of histamine receptors in the brain. It is quite common for people intolerant to histamine to suffer from fibromyalgia or diffuse pain that is difficult to explain. Histamine influences the release of serotonin and dopamine, so it is related to depression. Histamine influences blood pressure and heart rate, becoming a risk factor for heart disease as well. Add another factor, some people present polymorphisms to an enzyme fundamental in the synthesis of GABA, the main NeuroT with inhibitory effect on the central nervous system, this leads to a decrease in GABA production, with the alteration of the GABA/Glutamate ratio and consequently influences the basal activation state of the central nervous system. If we add the effects of the alteration of this mechanism to the hyperactivation by excess histamine, we find the exacerbation of the physical and psychic symptomatology connected to these phenomena [37].

In food [38], histamine is never alone, it is always accompanied by a considerable amount of other biogenic amines. This makes the symptoms and diagnosis of histamine intolerance complicated and sometimes rather unpredictable. Putrescine and Cadaverine in food can cause problems by interfering with AED activity, making it more difficult for us to degrade histamine in our intestines This can increase the level of histamine to which we are exposed The higher the level of putrescine and cadaverine in a food, the higher the actual level of histamine. Tyramine is degraded not by the AED, but by a different enzyme called monoamine oxidase (MAO). Tyramine is therefore known to cause problems in people taking antiquated antidepressants called "MAO-inhibitors", which block the destruction of tyramine. If tyramine accumulates, severe headaches and dangerous high blood pressure can occur. Here there is another important link, subjects with polymorphisms on MAO A and MAO B, are exposed to an altered effect of histamine, both for the symbiotic effect with other amines that are not degraded effectively and for the effect on the humoral tone due to the incorrect degradation of neurotransmitters; in fact, we have already seen how monoamine oxidase inhibitors have been found effective in psychiatric treatment [39] of panic disorder, even when associated with agoraphobia or social phobia, in the treatment of dysthymia, atypical depression, major depression, and bipolar depression, in suicidal risk, treatment of generalized anxiety, aggressive behavioral disorders, bulimia, obsessive-compulsive disorder, post-traumatic stress disorder, personality avoidance disorder, and borderline personality disorder [40-54].

\section{Conclusion}

Even if recent research confirms only the data already acquired in the literature, without any particular relevant news, the correlation of hyperhistaminosis with various physical and mental pathologies emerges, to the point of deserving more attention in the future. More and more emerges the direct relationship between body and mind and how these are interconnected. Hyperhistaminosis, besides being involved in immune, neurodegenerative, systemic vascular, cardiac, pulmonary, hepatic, kidney and skin diseases, is directly connected to a large part of mood disorders, depression, obsessive disorder, phobias, and various personality disorders, even if it is not yet clear whether the role of histamine is the main factor or cause that exacerbates the symptoms, also taking into account the neurobiological and genetic correlates related to the forms of hyperhistaminosis.

\section{References}

1. Kandel ER, James Schwartz, Thomas Jessel, Steven A Siegelbaum, Andrew J Hudspeth (2017) Principles of Neuroscience. Ambrosiana Publishing House, Italy.

2. Joan Izquierdo Casas, Oriol Comas Basté, M Luz Latorre Moratalla, Marian Lorente Gascón, Adriana Duelo, et al. (2018) Low serum diamine oxidase (DAO) activity levels in patients with migraine. J Physiol Biochem 74(1): 93-99.

3. Elena García Martín, Pedro Ayuso, Carmen Martínez, Miguel Blanca, José A G Agúndez (2009) Histamine pharmacogenomics. Pharmacogenomics 10(5): 867-883.

4. Elena García Martín, Carmen Martínez, Mercedes Serrador, Hortensia Alonso Navarro, Pedro Ayuso, et al. (2015) Diamine oxidase rs10156191 and rs2052129 variants are associated with the risk for migraine. Headache: The Journal of Head and Face Pain 55(2): 276-286.

5. Angelini C, Battistin L (2019) Clinical Neurology. Aesculapius Publishing Company, France.

6. Perrotta G (2019) Clinical Psychology, Luxco Ed, 1st edition, Italy.

7. Postle BR (2016) Cognitive Neuroscience. The essentials, Edra Ed.

8. Luo L (2017) Principles of Neurobiology. Zanichelli Ed, Italy.

9. M Cecchi, M B Passani, L Bacciottini, P F Mannaioni, P Blandina (2001) Cortical acetylcholine release elicited by stimulation of histamine $\mathrm{H} 1$ receptors in the nucleus basalis magnocellularis: a dual-probe microdialysis study in the freely moving rat. Eur. J. Neurosci 13(1): 6878.

10. Hofstra CL, Desai PJ, Thurmond RL, Fung Leung WP (2003) Histamine $\mathrm{H} 4$ receptor mediates chemotaxis and calcium mobilization of mast cells. J Pharmacol Exp Ther 305(3): 1212-1221.

11. M R Liebowitz, E Hollander, F Schneier, R Campeas, L Welkowitz, et al. (1990) Reversible and irreversible monoamine oxidase inhibitors in other psychiatric disorders. Acta Psychiatrica Scandinavica 82: (360): 29-34. 
12. Finberg JPM, Rabey JM (2016) Inhibitors of MAO-A and MAO-B, in Psychiatry and Neurology, in Frontiers in Pharmacology 7: PP 340.

13. Dowson JH (1987) MAO inhibitors in mental disease: their current status. Journal of Neural Transmission. [suppl]23: 121-138.

14. K Onodera , A Yamatodani, T Watanabe, H Wada (1994) Neuropharmacology of the histaminergic neuron system in the brain and its relationship with behavioral disorders. Prog Neurobiol 42(6): 685-702.

15. S J Hill, C R Ganellin, H Timmerman, J C Schwartz, N P Shankley, et al. (1997) International Union of Pharmacology. XIII. Classification of histamine receptors. Pharmacol Rev 49(3): 253-278.

16. Yong Xin Sun, Xiao Hong Wang, Ai Hua Xu, Jiu Han Zhao (2014) Functional polymorphisms of the MAO gene with Parkinson disease susceptibility: A meta-analysis. J Neurol Sc 345(1-2): 97-105.

17. Yoshiyuki Minegishi, Masako Saito, Tomohiro Morio, Ken Watanabe, Kazunaga Agematsu, et al. (2006) Human tyrosine kinase 2 deficiency reveals its requisite roles in multiple cytokine signals involved in innate and acquired immunity. Immunity 25(5): 745-755.

18. Laura Maintz, Natalija Novak (2007) Histamine and histamine intolerance. Am J Clin Nutr 85(5): 1185-1196.

19. Koro Gotoh, Takayuki Masaki, Seiichi Chiba, Keiko Higuchi, Tetsuya Kakuma, et al. (2009) Hypothalamic neuronal histamine signaling in the estrogen deficiency-induced obesity. J Neurochem 110(6): 1796-1805.

20. Peter D Arkwright, Mario Abinun, Andrew J Cant (2002) Autoimmunity in human primary immunodeficiency diseases. Blood 99(8): 2694-2702.

21. Patrick F K Yong, Alexandra F Freeman, Karin R Engelhardt, Steven Holland, Jennifer M Puck, et al. (2012) An update on the hyper-IgE syndromes. Arthritis Research \& Therapy 14(6): 228.

22. Marie-Olivia Chandesris, Isabelle Melki, Angels Natividad, Anne Puel, Claire Fieschi, et al. (2012) Autosomal Dominant STAT3 Deficiency and Hyper-IgE Syndrome. Medicine (Baltimore) 91(4): e1-19.

23. Milner JD, Brenchley JM, Laurence A, Alexandra F Freeman, Brenna Hill, et al. (2008) Impaired $\mathrm{T}(\mathrm{H}) 17$ cell differentiation in subjects with autosomal dominant hyper-IgE syndrome. Nature 452(7188): 773-776.

24. Cindy S Ma, Danielle T Avery, Anna Chan, Marcel Batten, Jacinta Bustamante, et al. (2012) Functional STAT3 deficiency compromises the generation of human T follicular helper cells. Blood 119(17): 39974008.

25. Vibhav Sekhsaria, Lori E Dodd, Amy P Hsu, Jennifer R Heimall, Alexandra F Freeman, et al. (2011) Plasma metalloproteinase levels are dysregulated in signal transducer and activator of transcription 3 mutated hyper-IgE syndrome. J Allergy Clin Immunol 128(5): 1124-1127.

26. Jing Wu, Ji Chen, Zhi Qing Tian, Hao Zhang, Ruo Lan Gong, et al. (2017) Clinical Manifestations and Genetic Analysis of 17 Patients with Autosomal Dominant Hyper-IgE Syndrome in Mainland China: New Reports and a Literature Review. J Clin Immunol 37(2): 166-179.

27. Karin R Engelhardt, Sean McGhee, Sabine Winkler, Atfa Sassi, Cristina Woellner, et al. (2009) Large Deletions and Point Mutations Involving dedicator of cytokinesis 8 (DOCK8) in the Autosomal Recessive Form of the Hyper-IgE Syndrome, J Allergy Clin Immunol 124(6): 1289-1302.

28. Borges WG, Augustine NH, Hill HR (2000) Defective interleukin-12/ interferon-gamma pathway in patients with hyperimmunoglobulinemia E syndrome. J Pediatr 136(2): 176-80.

29. Martins MN, Tullu MS, Mahajan SA (2001) Molluscum contagiosum and Job's syndrome. J Postgrad Med 47(4): 268-269.

30. Linlin Yang, Manfred Fliegauf, Bodo Grimbacher, et al. (2014) Hyper-IgE syndromes: reviewing PGM3 deficiency. Curr Opin Pediatr 26(6): 697703.
31. Yoshiyuki Minegishi, Masako Saito, Masayuki Nagasawa, Hidetoshi Takada, Toshiro Hara, et al. (2009) Molecular explanation for the contradiction between systemic Th17 defect and localized bacterial infection in hyper-IgE syndrome. J Exp Med 206(6): 1291-1301.

32. Andrea M Siegel, Kelly D Stone, Glenn Cruse, Monica G Lawrence, Ana Olivera, et al. (2013) Diminished allergic disease in patients with STAT3 mutations reveals a role for STAT3 signaling in mast cell degranulation. J Allergy Clin Immunol 132(6): 1388-1396.

33. Luca Esposito, Laura Poletti, Cinzia Maspero, Alessandro Porro, Maria Cristina Pietrogrande, et al. (2012) Hyper-IgE syndrome: dental implications. Oral Surg Oral Med Oral Pathol Oral Radiol. 114(2): 147153.

34. Alexandra F Freeman, Elizabeth Mannino Avila, Pamela A Shaw, Joie Davis, Amy P Hsu, et al. (2011) Coronary artery abnormalities in HyperIgE syndrome. J Clin Immunol. 31(3): 338-345.

35. Masakatsu Yanagimachi, Takashi Ohya, Tomoko Yokosuka, Ryosuke Kajiwara, Fumiko Tanaka, et al. (2016) The Potential and Limits of Hematopoietic Stem Cell Transplantation for the Treatment of Autosomal Dominant Hyper-IgE Syndrome. J Clin Immunol 36(5): 511-516.

36. R E Brown, D R Stevens, H L Haas (2001) The physiology of brain histamine. Prog Neurobiol 63: 637-672.

37. Prell GD, Green JP (1986) Histamine as a Neuroregulator. Annu Rev Neurosci 9: 209-254.

38. Ke Yong Wang, Akihide Tanimoto, Sohsuke Yamada, Xin Guo, Yan Ding, et al. (2015) Histamine Regulation in Glucose and Lipid Metabolism via Histamine Receptors. Am J Pathol, 177(2): 713-723.

39. American Psychiatric Association (2013) Diagnostic and Statistical Manual of Mental Disorders, Fifth Edition, USA.

40. Alan G Mallinger, Ellen Frank, Michael E Thase, Michelle M Barwell, Nancy Diazgranados, et al. (2009) Revisiting the Effectiveness of Standard Antidepressants in Bipolar Disorder: Are Monoamine Oxidase Inhibitors Superior? Psychopharmacology bulletin 42(2): 64-67.

41. Lisheng Du, David Bakish, Arun Ravindran, Pavel D Hrdina (2004) MAO-A gene polymorphisms are associated with major depression and sleep disturbance in males. Neuroreport 15(13): 2097-2101.

42. Ho Jang Kwon, Han Jun Jin, Myung Ho Lim (2014) Association Between Monoamine Oxidase Gene Polymorphisms and Attention Deficit Hyperactivity Disorder in Korean Children. Genet Test Mol Biomarkers 18(7): 505-509.

43. Volavka J, Bilder R, Nolan K (2010) Catecholamines and Aggression: The Role of COMT and MAO Polymorphisms 1036(1): 393-398.

44. Aline Santos Sampaio, Ana Gabriela Hounie, Kátia Petribú, Carolina Cappi, Ivanil Morais, et al. (2015) COMT and MAO-A Polymorphisms and Obsessive-Compulsive Disorder: A Family-Based association study. PloS One 10(3): e0119592.

45. Perrotta G (2019) Anxiety disorders: definitions, contexts, neural correlates, and strategic therapy. Jacobs Journal of Neurology and Neuroscience 6(1): 046.

46. Perrotta G (2019) Depressive disorders: Definitions, contexts, differential diagnosis, neural correlates, and clinical strategies. Archives of Depression and Anxiety 5(2): 009-033.

47. Perrotta G (2019) Post-traumatic stress disorder: Definition, contexts, neural correlations, and cognitive-behavioral therapy. Journal of Public Health and Nutrition 2(2): 40-47.

48. Perrotta G (2019) Psychological trauma: definition, clinical contexts, neural correlations and therapeutic approaches. Current Research in Psychiatry and Brain Disorders. Curr Res Psychiatry Brain Disord: CRPBD-100006. 
49. Perrotta G (2019) Panic disorder: definitions, contexts, neural correlates and clinical strategies. Current Trends in Clinical \& Medical Sciences, Curr Tr Clin \& Med Sci. 1(2): PP: 1-10.

50. Perrotta G (2019) Sleep-wake disorders: Definition, contexts and neural correlations. J Neurol Psychol 7(1): 1-11.

51. Perrotta G (2019) Obsessive-Compulsive Disorder: definition, contexts, neural correlates and clinical strategies. Scientific Journal of Neurology 1.4: 08-16.
52. Perrotta G (2020) Suicidal risk: definition, contexts, differential diagnosis, neural correlates and clinical strategies. J Neuroscience and Neurological Surgery, 6(2): Pp 114.

53. Perrotta G (2019) General Psychology, Luxco Ed, 1st ed, Italy.

54. Perrotta G (2020) The strategic clinical model in psychotherapy: theoretical and practical profiles. Journal of Addiction and Adolescent Behaviour 3(1): 1-5.

\section{(c) (i)}

To Submit Your Article Click Here:

Submit Article
DOI: $10.32474 / 0 J N B D .2020 .04 .000187$

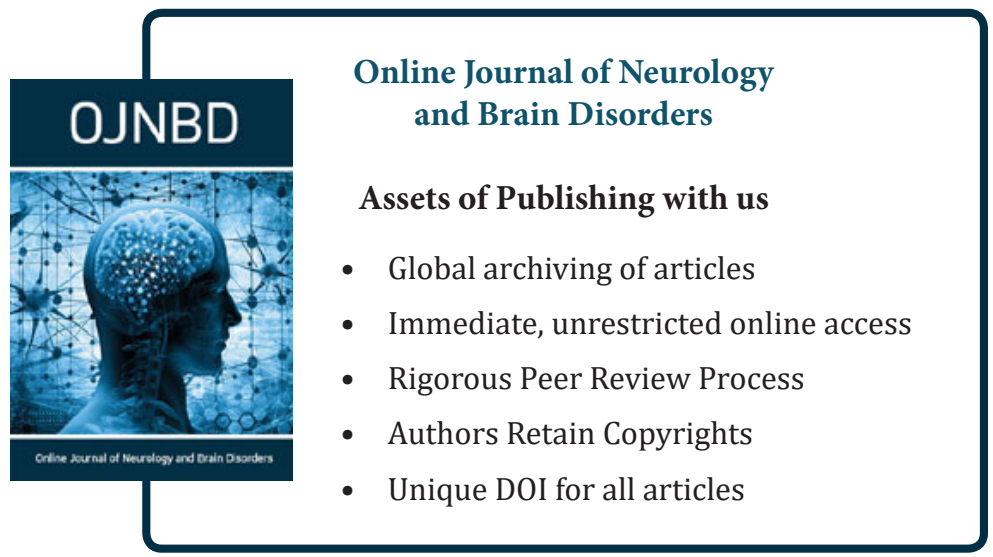

